\title{
LA PUESTA EN VALOR DEL MANTO BLANCO DE PARACAS: UN PROYECTO DE GESTIÓN DE UN PATRIMONIO CULTURAL SANMAROUINO
}

\author{
CÉsAR Augusto Franco TORRES \\ UNIVERSIDAD NACIONAL MAYOR DE SAN MARCOS \\ cefranto@yahoo.es
}

\section{RESUMEN}

El año 2016 el Museo de Arqueología y Antropología de San Marcos y el Centro Cultural inauguraron, con motivo del 465 aniversario de la Universidad, la exposición museográfica: "Sala Paracas: Vientos del Sur", la cual significó la recuperación y puesta en valor del Manto Blanco de Paracas, un bien patrimonial de nuestra universidad, único en su género, por sus características peculiares, tanto de manufactura como decorativas.

Palabras claves: patrimonio cultural, arqueología, Paracas, Universidad San Marcos.

\section{Abstract}

In 2016 the Museo de Arqueología y Antropología de San Marcos and the Centro Cultural inaugurated, on the occasion of the 465th anniversary of the University, the museographic exhibition: " Sala Paracas: Vientos del Sur “, which meant the recovery and valorization of the White Mantle of Paracas, an asset of our university, unique in its kind, for its peculiar characteristics, both of manufacturing and decorative.

KeYwords: cultural heritage, archeology, Paracas, San Marcos University.

\section{INTRODUCCIÓN}

Presentamos en el presente artículo, el proceso seguido para la recuperación y puesta en valor del Manto Blanco de la cultura Paracas, que forma parte de las colecciones arqueológicas del Museo de Arqueología y Antropología de la Universidad Nacional Mayor de San Marcos. Se trata de un manto con particularidades culturales, tanto en su manufactura como en su conjunto de motivos iconográficos, no hallado en algún otro textil de la cultura paracas, ni en otra cultura andina prehispánica. 
La Universidad custodia celosamente miles de bienes culturales, tanto arqueológicos -que fueron recuperados por el Dr. Julio C. Tello en sus múltiples investigaciones por todo el territorio nacional, como por posteriores investigadores (docentes y estudiantes) de nuestra casa de estudios-, bienes históricos como la Bula Papal y la Real Cédula de Fundación de nuestra universidad, y bienes artísticos.

Por ello, nuestras autoridades avalaron la recuperación y puesta en valor de esta pieza emblemática de la cultura Paracas, como es el manto Blanco, para que toda la comunidad nacional, pueda observarlo con detalle, tomando atención de sus características inigualables.

\section{El Manto Blanco de Paracas}

La cultura Paracas se desarrolló en el periodo Formativo tardío en el actual departamento de Ica (Lazo; 2016), extendiéndose altitudinalmente hasta los departamentos de Huancavelica y Ayacucho. Los Paracas fueron uno de los mejores tejedores de toda América prehispánica, sobresaliendo sus hermosos textiles, de alta tecnología de manufactura (Aponte; 2016).

El Manto Blanco de la cultura Paracas, es un textil único en su género por sus características peculiares. Se trata de un textil de la fase Paracas Tardío Tansicional (200 a.C. - 100 a. C.), por sus características estilísticas y decorativas (Bueno, 2016: 32). Es de forma rectangular, de 278 centímetros de largo por 138 centímetros de ancho, conformado por tres ejes (un paño central y dos paños laterales) y los flecos (van Dalen e Yntusca, 2016). El textil está elaborado en fibra de camélidos y algodón. En la parte central se encuentran 120 personajes (de sexo femenino y masculino), los cuales corresponden a tres personajes femeninos y cinco masculinos que se repiten (Chocano, 2012).

Van Dalen e Yntusca (2016) han identificado tres fases de elaboración del manto: Fase 1: Elaboración y unión de los tres paños, conformándose una unidad textil. Reforzamiento de los paños mediante el refuerzo por trama. Fase 2: decoración de los tres paños mediante la técnica de bordado en tres variantes: punto atrás, punto de relleno y punto doble cruz. Fase 3: unión final de las telas base mediante la técnica punto surgete, tejido de dos tiras de flecos y unión de los flecos al manto mediante anillado cruzado.

Este manto fue adquirido por el sabio Julio C. Tello durante sus investigaciones en el departamento de Ica, desconociéndose exactamente el lugar de procedencia del mismo; depositándolo luego, en los ambientes del Museo de San Marcos, hoy Museo de Arqueología y Antropología de la Universidad.

\section{El Proyecto de Puesta en Valor del Manto Blanco de Paracas}

Durante gran parte del siglo XX el manto Blanco de paracas se encontró confundido y refundido con el resto de objetos y materiales culturales que forman parte del Museo de San Marcos, en sus depósitos. En la primera mitad de la década de 1970, siendo director del museo el Dr. Luis G. lumbreras, se realizó una exposición museográfica, donde se presentó el Manto Blanco de Paracas a la comunidad académica.

El año 2004, con apoyo del Banco de Crédito del Perú (BCP), como parte del premio Fondo del Embajador, se obtuvo una urna de madera con cobertura de vidrio, a fin de albergar en su interior de forma extendida (antes se encontraba doblado) el Manto Blanco de Paracas.

El año 2016, con la gestión transitoria de la Rectora de la Universidad Dra. Antonia Castro, bajo la iniciativa y monitoreo del Director General de Centro Cultural de la UNMSM Ing. Cesar A. Franco Torres se inició en el mes de febrero el proyecto Recuperación y Puesta en Valor del manto Blanco de Paracas, que tenía como objetivo principal la recuperación, conservación y exposición de este importante patrimonio cultural de la universidad y de todos los peruanos. 
Para ello se desarrolló el proyecto museográfico a cargo del responsable del área de museografía del museo, Lic. Juan Yataco Capcha, el Director del Museo de Arqueología y Antropología de San Marcos, Lic. Pieter van Dalen Luna, el responsable de la oficina de restauración Arq. Martín Fabbri y el encargado de Operaciones Generales y Mantenimiento del Centro Cultural, Ing. Pedro Ramírez. El proyecto de exposición se desarrolló en la Sala de exposición 1, donde se hallaba una exposición permanente desde el año 2004 titulado: "Los Orígenes".

\section{Equipos y Medidas de Seguridad Utilizados}

El proyecto museográfico: "Sala Paracas: Vientos del Sur", supuso la habilitación de equipos y sistemas integrados en la sala de exposición, que vayan acorde con la importancia de los bienes a exponer. Debido a que la exposición iba a estar dirigida no solo a la comunidad académica, sino a la comunidad en general, se optó por utilizar en las descripciones una temática específica con un lenguaje claro y sencillo (Yataco, 2016: 2-10). La sala de exposición cuenta con dos espacios: el primero con un área de $146.305 \mathrm{~m}^{2}$ y el segundo con un área de $37.6887 \mathrm{~m}^{2}$. Toda esta sala cuenta con un sistema de circuito cerrado de cámaras de seguridad, con cuatro detectores de movimiento y cuatro cámaras de seguridad instalados en las salas, así como detectores de humo. Para el acceso a salas se debe contar con una tarjeta electrónica, con el cual se abren dos puertas electrónicas.

Se ha instalado también, controladores de humedad que permitan mantener al Manto Blanco dentro de un rango de 55\% y 60\% y temperatura al interior de la sala, para monitorear la temperatura ambiental y evitar que los bienes culturales sufran algún tipo de daño por el incremento de la temperatura ambiental, la cual está controlada con un sistema de refrigeración; pues los textiles en exposición, deben de estar en temperaturas no menor de $18^{\circ}$ y no mayor a $21^{\circ} \mathrm{C}$. Por ello se instaló dos termo-higrómetros localizados en dos extremos opuestos de la sala de exposición; así como cuatro termo-higrómetros de mano de marca Col Boox, colocados cada uno al interior de las vitrinas que contienen los materiales orgánicos. Así mismo para evitar la exposición indebida del Manto Blanco a la iluminación y garantizar su cuidado y sostenibilidad, se instaló un sistema PLC que permite programar la iluminación en las diferentes estaciones, evitando que el manto blanco se exponga a la iluminación un tiempo mayor a $4 \mathrm{mn}$ dentro de un ciclo de $18 \mathrm{mn}$.

Se clausuraron mediante el tapiado, otras puertas y ventanas de acceso a la sala de exposición, a fin que el único acceso sea por la puerta eléctrica, esto como medida de seguridad. Previo a todos los trabajos, se cambió el piso de parquet en la sala de exposición, pues este se hallaba deteriorado por el paso de los años y la humedad ambiental.

Para contrarrestar la acción de la iluminación en los materiales arqueológicos, en especial en los textiles, se instaló cuarenta y cuatro luces leds en los carriles aéreos de las dos salas de exposición. Los juegos de spots de luces fueron reciclados de antiguos dicroicos y fueron cambiados al sistema de dicroicos leds. Se instaló luces leds con filtros UV en tres vitrinas de exposición que contenían a los textiles Paracas. Estas medidas se adoptaron para evitar el deterioro de las fibras textiles. El recorrido de las secuencias de exposición presenta encendido automático no superior a los tres minutos. La iluminación del manto blanco tiene 50 luxes; esto a fin de conservarlo.

\section{Conservación de los Materiales Arqueológicos en Exposición}

Antes de la exposición, todas las piezas seleccionadas de la cultura paracas, fueron diagnosticadas, en cuanto a su estado de conservación, aplicando procesos de conservación preventivo. De igual manera, para la conservación de los textiles, se contó con la presencia de un equipo de conservadores 
conformado por la Lic. Maribel Medina y Carmen Malásquez, quienes durante dos meses realizaron trabajos de fijación, consolidación y retiro de agentes ajenos a los textiles.

\section{Montaje De las Salas}

Entre el 25 al 29 de abril se realizó las labores de montaje de salas, para lo cual ya se encontraban instalados los sistemas de electricidad, aire acondicionado, entre otros. Se instaló siete vitrinas en las salas de exposición. Se utilizó en la exposición 14 piezas ( 9 piezas cerámicas, 1 lítico, 3 textiles y 1 cuerpo incompleto de momia de sexo femenino), las cuales presentan un estado de conservación y estabilidad óptima para su exposición.

\section{INAUGURACIÓN DE LA EXPOSICIÓN}

El día 11 de mayo de 2016, al inicio de la semana de celebraciones por el 465 aniversario de la Universidad Nacional Mayor de San Marcos, en horas del mediodía y con presencia de todas las autoridades universitarias, y la representante de la UNESCO en el Perú, se inauguró la sala de exposición "Paracas: Vientos del Sur".

\section{Conclusiones}

Después de mucho tiempo se ha realizado un proyecto que permite poner en valor un patrimonio cultural de la Universidad Nacional Mayor de San Marcos, como es el Manto Blanco de Paracas. Esta exposición permite que la comunidad nacional en general, pueda admirar un importante textil con su belleza en colores, en motivos y en tecnología de elaboración. Los diseños que presenta son representativos de la cultura Paracas, la cual justamente sobresalió entre todas las culturas andinas prehispánicas por su textilería y patrones funerarios.

\section{BiBLIOGRAFÍA}

APONTE, Delia

2016 “La colección textil Paracas Necrópolis del Museo de Arqueología y Antropología de la Universidad Nacional Mayor de San Marcos". Colección Paracas. Joyas Sanmarquinas. Museo de Arqueología y Antropología de San Marcos, Centro Cultural, Universidad Nacional Mayor de San Marcos. Lima, pp. 37-60.

BUENO MENDOZA, Alberto

2016 "Paracas: cultura formativa del sur medio del Perú". Colección Paracas. Joyas Sanmarquinas. Museo de Arqueología y Antropología de San Marcos, Centro Cultural, Universidad Nacional Mayor de San Marcos. Lima, pp. 19-36.

CHOCANO MENA, Lourdes

2012 "Análisis de los personajes de un tejido paracas: una interpretación iconográfica del manto Blanco". Arqueología y Sociedad № 24. Museo de Arqueología y Antropología de San Marcos, Centro Cultural, Universidad Nacional Mayor de San Marcos. Lima, pp. 227-248.

FABBRI, Martín

2016 "Informe final de la Sala de exposiciones de arqueología". Proyecto expo Manto Paracas. Lima, 11 pp. 
LAZO PÉREZ, Roy

2016 “Introducción al estudio del territorio Paracas". Colección Paracas. Joyas Sanmarquinas. Museo de Arqueología y Antropología de San Marcos, Centro Cultural, Universidad Nacional Mayor de San Marcos. Lima, pp. 11-18.

RAMÍREZ ESPINOZA, Pedro

2016 Implementación de la Sala de Exhibición Manto Blanco de Paracas. Centro Cultural de San marcos. Lima, 263 pp.

VAN DALEN LUNA, Pieter

2016 “Informe sobre el proyecto museográfico de la Sala Paracas, Vientos del Sur". Museo de Arqueología y Antropología de San Marcos. Lima, 34 pp.

VAN DALEN LUNA, Pieter e YNTUSCA GARCÍA, Andrés

2016 “¿Cómo se elaboró el Manto Blanco de Paracas Necrópolis? Proceso de manufacturación y tecnología textil aplicada”. Colección Paracas. Joyas Sanmarquinas. Museo de Arqueología y Antropología de San Marcos, Centro Cultural, Universidad Nacional Mayor de San Marcos. Lima, pp. 61-92.

YATACO CAPCHA, Juan

2016 Informe Técnico de Museografía. Sala Paracas: Vientos del Sur. Museo de Arqueología y Antropología de San Marcos. Universidad Nacional Mayor de San Marcos. Lima, 36 pp.

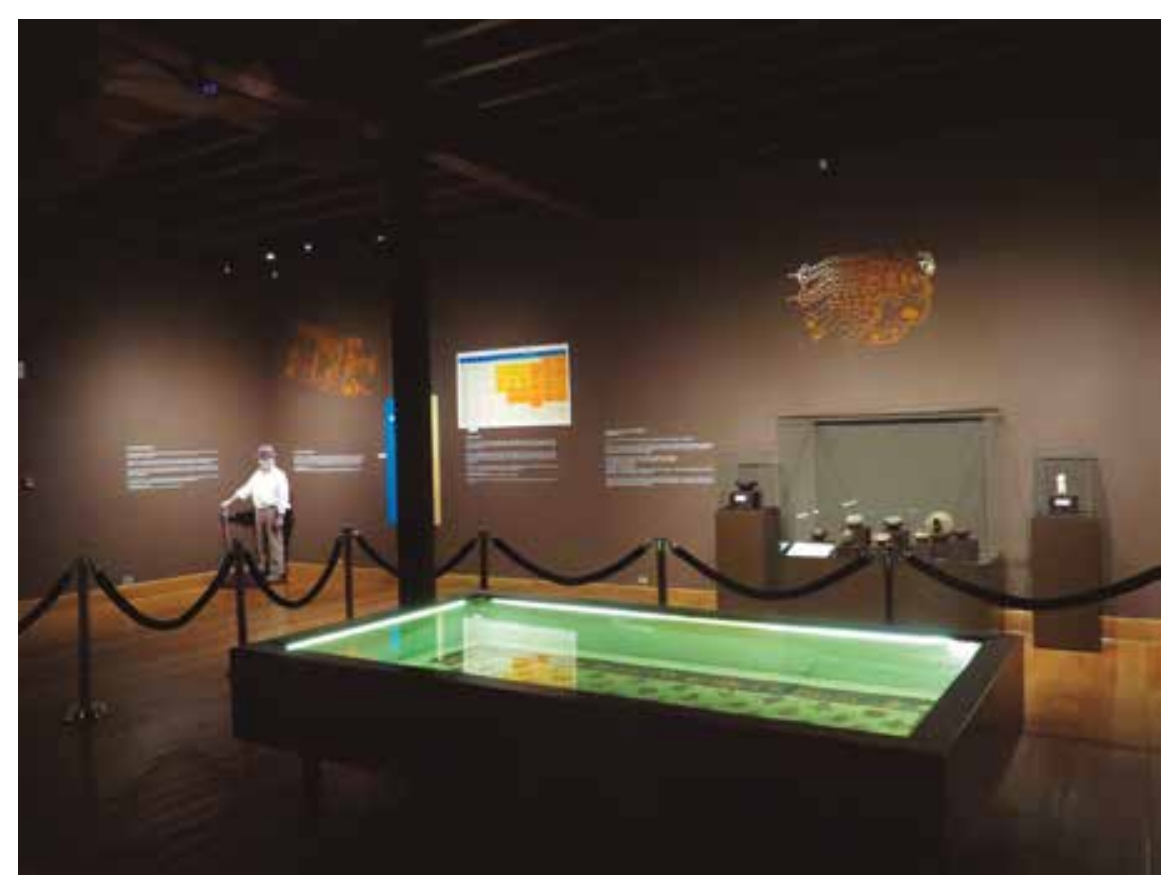

Figura 1: Vista de la sala de exposición. 


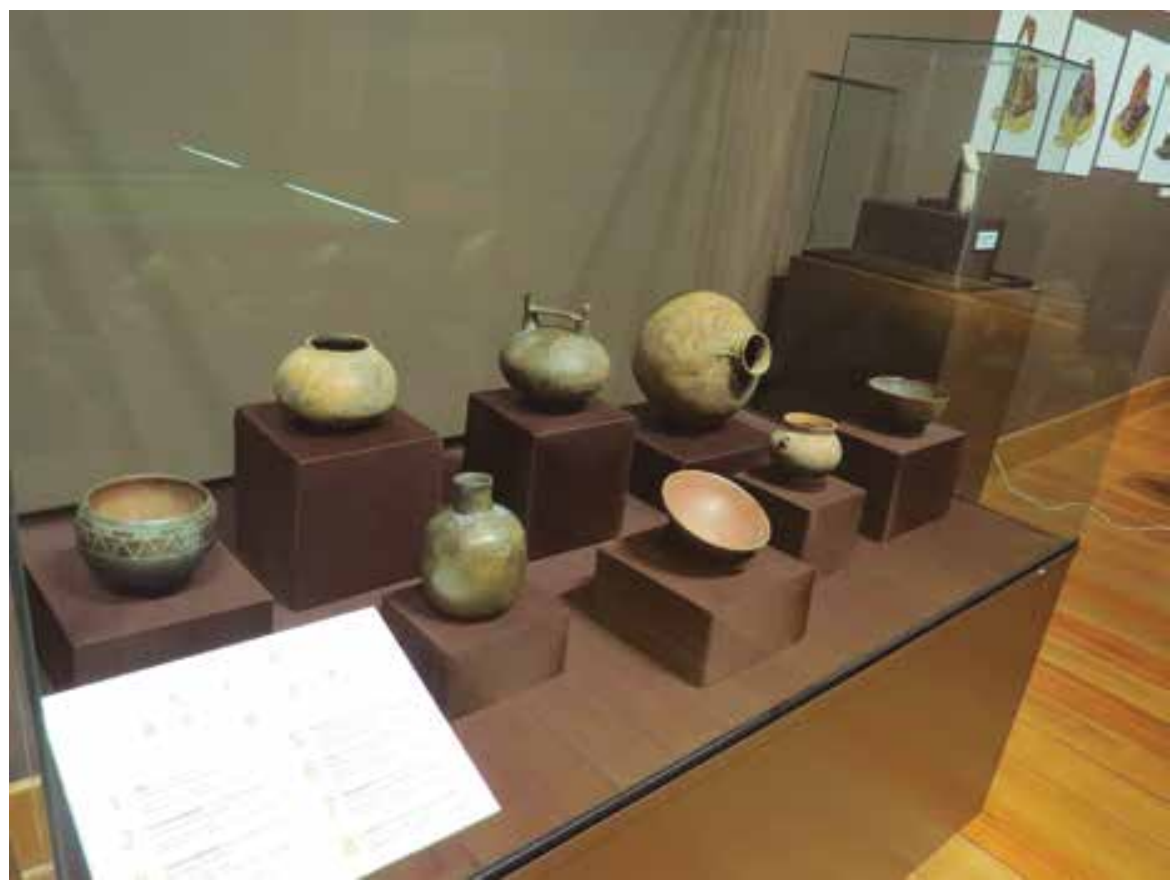

Figura 2: Vista de las vasijas cerámicas de la cultura Paracas.

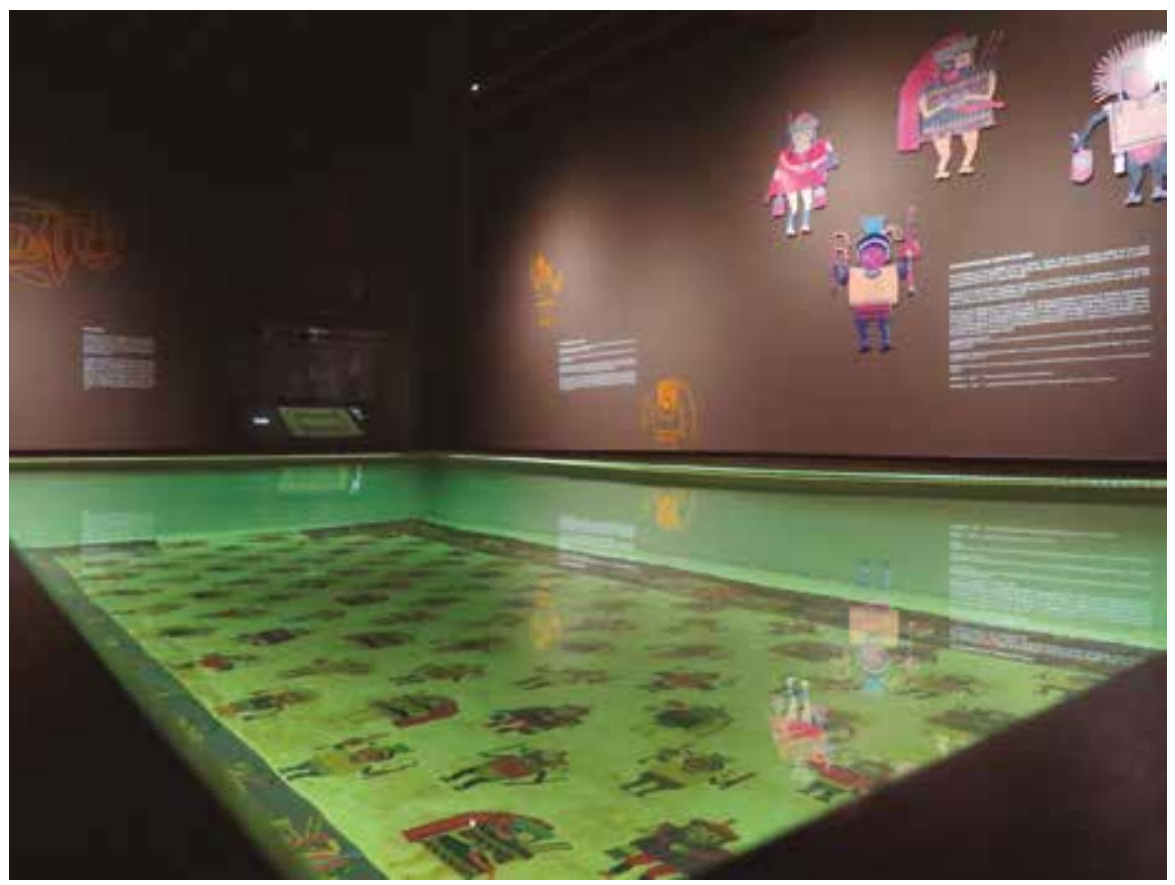

Figura 3: El Manto Blanco de Paracas ha recibido tratamientos adecuados de conservación para su puesta en valor. 